\title{
Getting the Most out of Direct Detection Cameras for Low-Dose Transmission Electron Microscopy
}

\author{
Anchi Cheng ${ }^{1}$, James Pulokas ${ }^{1}$, Sargis Dallakyan ${ }^{1}$, Amber Herold ${ }^{1}$, Clinton S. Potter ${ }^{1}$, Bridget \\ Carragher $^{1}$ \\ ${ }^{1}$ National Resource for Automated Molecular Microscopy (NRAMM), Department of Integrative \\ Structural and Computational Biology, The Scripps Research Institute, La Jolla, CA, USA
}

Single-particle cryo electron microscopy (cryoEM) is undergoing a technical revolution due to the recent developments of direct detectors. These new recording devices detect electrons directly (i.e. without conversion into light) and feature significantly improved detective quantum efficiency (DQE) as compared to photographic films or CCDs. Moreover, their enhanced readout rates allow for the collection of data as movies composed of many frames, during the time lapse previously corresponding to a single exposure. This in turn allows for correcting for beam-induced sample movement, a factor that has until now limited the resolution attainable using cryoEM.

There are currently three commercial providers of direct detectors and at NRAMM, we have had access to devices from each of these companies (DE12 from Direct Electron, K2 Summit from Gatan, and Falcon II from FEI). We have acquired low-dose ( 20-50 electrons/ $\left./ \AA^{2}\right)$ images at a rate of 500-1000 images (or movies) per day using our automated data acquisition software Leginon [1]. This rate of data accumulation requires careful management of resources and near real-time processing. We use a series of tools implemented in Appion [2] to manage the data and align the movie frames asynchronously. This provides an efficient pipeline for routine and sustained use of DD's.

This workshop presentation will introduce the principles of direct electron detection and highlight the similarities and differences between the three devices we have used. Specific data collection strategies optimized for each device will be discussed. 


\section{References:}

[1] C. Suloway et al, J. Struct. Biol. 151 (2005) p. 41-60 [2] G.C.

Lander et al, J. Struct. Biol. 16 (2009) p. 95-102

[3] The authors acknowledge funding from the National Institutes of Health (GM103310).

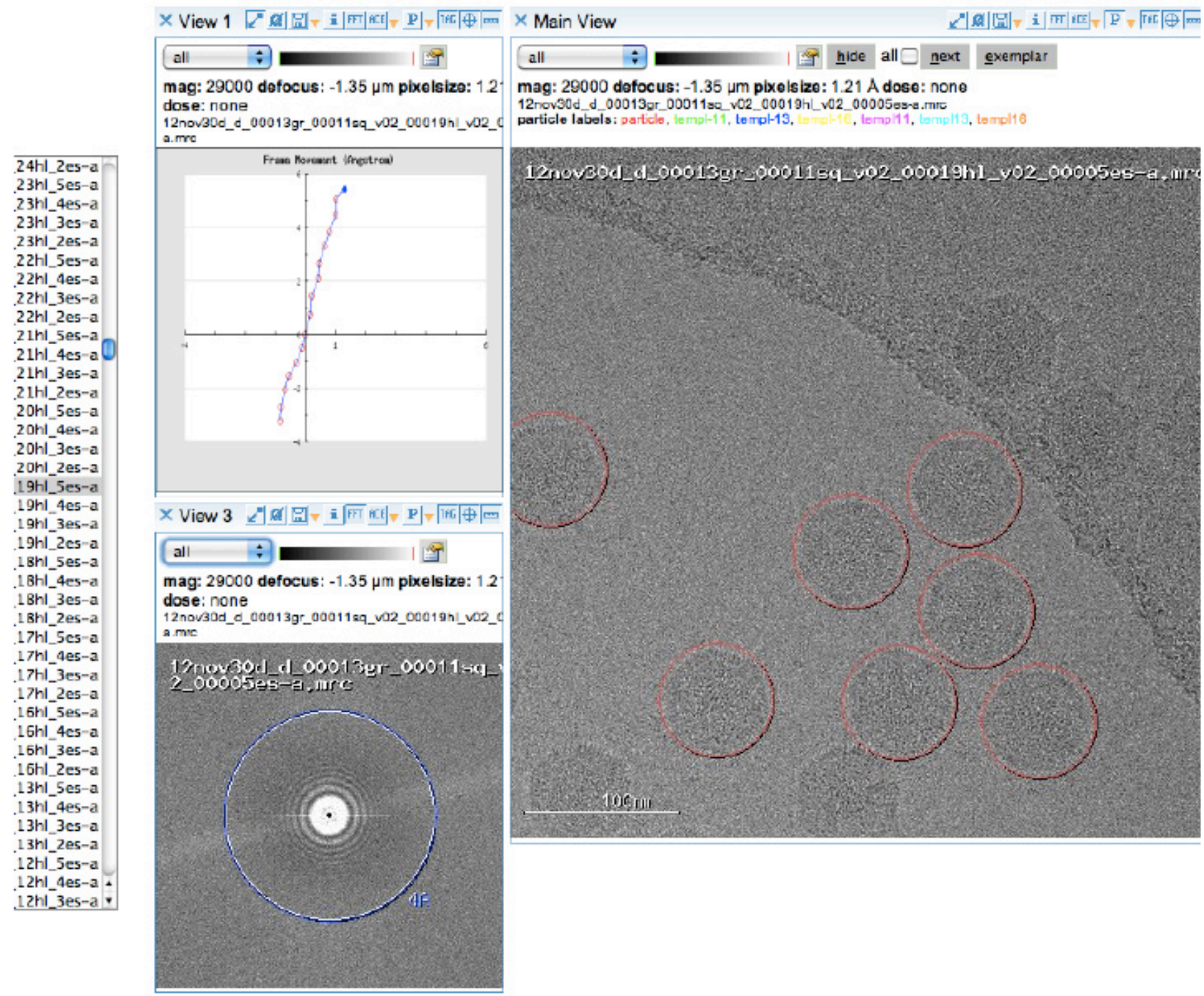

Figure 1. Leginon/Appion image viewer showing frame-aligned images (Main View) along with the shifts measured by the alignment algorithm (View 1) and power spectrum (View 3) of the image.The frame-aligned images are immediately available for Appion image processing just like those acquired by non-frame saving camera, providing a straightforward transition for the users. 